\title{
Tip-Enhanced Fluorescence Microscopy at 10 Nanometer Resolution
}

\author{
Jordan M. Gerton, ${ }^{*}$ Lawrence A. Wade, Guillaume A. Lessard, Z. Ma, and Stephen R. Quake \\ Department of Applied Physics, California Institute of Technology, MC 128-95, Pasadena, California 91125, USA
}

(Received 12 December 2003; published 28 October 2004)

\begin{abstract}
We demonstrate unambiguously that the field enhancement near the apex of a laser-illuminated silicon tip decays according to a power law that is moderated by a single parameter characterizing the tip sharpness. Oscillating the probe in intermittent contact with a semiconductor nanocrystal strongly modulates the fluorescence excitation rate, providing robust optical contrast and enabling excellent background rejection. Laterally encoded demodulation yields images with $<10 \mathrm{~nm}$ spatial resolution, consistent with independent measurements of tip sharpness.
\end{abstract}

DOI: 10.1103/PhysRevLett.93.180801

PACS numbers: 07.79.Fc, 42.50.Hz, 61.46.+w, 78.67.Bf

The potential of near-field microscopy to optically resolve structure well below the diffraction limit has excited physicists, chemists, and biologists for almost 20 years. Conventional near-field scanning optical microscopy (NSOM) uses the light forced through a small metal aperture to locally excite or detect an optical response. The spatial resolution in NSOM is limited to 30$50 \mathrm{~nm}$ by the penetration depth of light into the metal aperture. More recently, apertureless-NSOM (ANSOM) techniques were developed which leverage the strong enhancement of an externally applied optical field at the apex of a sharp tip for local excitation of the sample [111]. The promised advantage of ANSOM is that spatial resolution should be limited only by tip sharpness (typically $\sim 10 \mathrm{~nm}$ ). The resolution in most previous ANSOM experiments, however, was at best marginally better than NSOM and was inferior to expectations based on tip sharpness alone. Further, the external field used to induce enhancement led to a substantial background signal and to assertions that one-photon fluorescence is not appropriate for ANSOM $[12,13]$. These experiments fell short of their potential because they maintained a tip-sample gap of several nanometers, and thus did not thoroughly exploit the tightly confined enhancement.

Here, we demonstrate an ANSOM technique that fully exploits the available contrast and leads to spatial resolution that is limited only by tip sharpness. The problems associated with a tip-sample gap are overcome by oscillating the probe in intermittent contact with the sample. The detected signal is then composed of a modulated near-field portion that is superimposed on the far-field background. Subsequent demodulation decouples the two components and thus strongly elevates the near-field signal relative to the background. With this technique, we measured $<10 \mathrm{~nm}$ lateral resolution via one-photon fluorescence imaging of isolated quantum dots, consistent with independent measurements of tip sharpness. The measured resolution is $>3$ times better than previous reports for quantum dots using one-photon fluorescence $[8,9]$, and is $\sim 2$ times better than previous measurements using higher-order optical processes (two-photon fluorescence [6], Raman scattering [4,5]) despite predictions to the contrary $[12,13]$.

To better understand the advantages of this technique and to facilitate development of accurate physical models, it is crucial to rigorously characterize the spatial confinement of the enhancement effect. Previous investigations did not attain the level of precision necessary to differentiate between various theoretical models $[4,7,14]$ and there was no experimental or theoretical consensus regarding either the functionality or the set of parameters governing the spatial confinement [12,15-18]. In this Letter, we show unambiguously that the enhancement decays strictly according to a power-law functionality moderated by a single parameter that characterizes the tip sharpness. The collective results shown here will impact not only nano-optics research, but also the application of ANSOM to a wide range of nanoscale systems at the interface between physics, biology, and chemistry.

Our instrument combines a custom optical layout with a commercial (Digital Instruments Bioscope) atomic force microscope (AFM). The excitation laser beam is focused through a glass coverslip (spot size: $350 \times 1000 \mathrm{~nm}$ ) using a 1.3 N.A. oil-immersion objective lens. The objective simultaneously collects fluorescence, which is then directed onto an avalanche photodiode through a system of spectral filters (background rejection-ratio $\sim 10^{10}: 1$ ). A beam-shaping mask is inserted into the excitation beam to generate a purely evanescent field above the glass interface (decay length: $120-250 \mathrm{~nm}$ ) with a large polarization component along the probe axis [19] as required for field enhancement $[15,20]$. The focal spot is aligned with the AFM probe by means of a piezoactuated tip-tilt mirror, and the lateral position of the probe is controlled by closed-loop feedback. Uncorrected residual drift $(0.05-0.2 \mathrm{~nm} / \mathrm{s})$ is the dominant source of uncertainty in the probe position.

The silicon AFM probe oscillates [21] with a typical peak-peak amplitude of 30-40 nm, assuring tip-sample contact at closest approach. In contrast, previous work 
used very small oscillations $(\sim 1 \mathrm{~nm})[7,8]$ or shear-force feedback $[4,6,10,14]$ to maintain a tip-sample gap of several nanometers. Data are recorded to a computer disk in the form of two independent streams of time stamps: one that marks the arrival time of each detected photon, and one that marks a particular phase in each probe-oscillation cycle. Because the raw data are stored permanently in a lossless digital format, multiple analysis algorithms can be applied without degrading the signal.

Samples are prepared by drying a dilute solution of $\mathrm{CdSe}-\mathrm{ZnS}$ core-shell quantum dots onto a clean glass coverslip. The dots have a mean diameter of $\sim 5 \mathrm{~nm}$ and an emission spectrum centered near $\lambda=600 \mathrm{~nm}$. The fluorescence rate is highly dynamic, exhibiting "blinking" and sudden changes in quantum yield (QY), in agreement with previous observations $[8,22,23]$. When a quantum dot is "on" and in a high QY state, a typical count rate of $\sim 2 \times 10^{4} \mathrm{sec}^{-1}$ is measured with $\sim 300 \mathrm{nW}$ of illumination power.

To determine which parameters influence the tipenhanced intensity distribution we measure the induced fluorescence rate as a function of tip-sample separation (z). The focal spot and AFM probe are centered on an isolated dot and the photon and probe-oscillation data streams are recorded for several seconds. A histogram of the phase delay $(\Delta)$ between the arrival time of each photon and the preceding probe-oscillation time stamp is computed. Each value of $\Delta$ is then mapped to the corresponding value of $z$ to produce an approach curve (Fig. 1). Each approach curve is a convolution of the tip-enhanced intensity distribution and the excitation-probability dis-

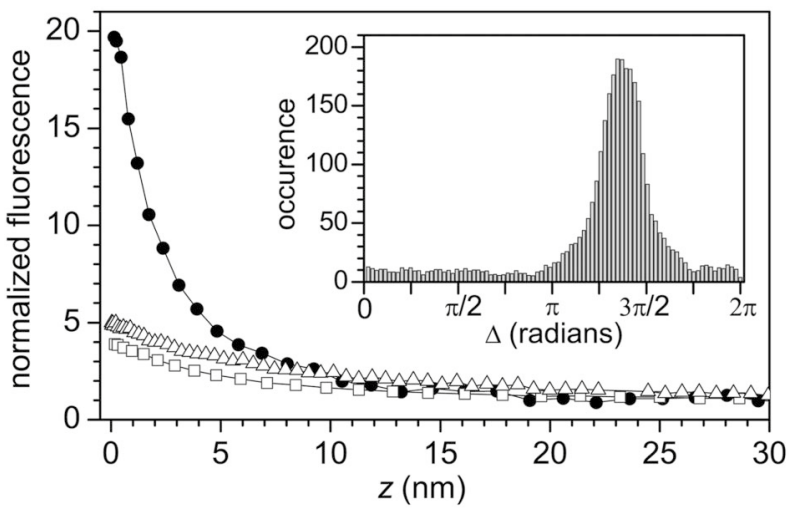

FIG. 1. Enhancement near a sharp silicon probe. Approach curves for a $5 \mathrm{~nm}$ diameter quantum dot (solid circles), a $5 \times$ $20 \mathrm{~nm} \mathrm{CdSe-ZnS} \mathrm{nanorod} \mathrm{(open} \mathrm{squares),} \mathrm{and} \mathrm{a} 20 \mathrm{~nm}$ diameter dye-doped latex sphere (open triangles). Additional approach curves extending to $z \sim 150 \mathrm{~nm}$ (not shown) were flat beyond the enhancement region. The vertical scale is normalized to the count rate for a retracted probe. $10 \%$ uncertainties in the horizontal and vertical scales originate from calibration of the probe-oscillation amplitude and noise in the normalization factor, respectively. The lines connect the data points. Inset: Histogram of phase delays for the quantum dot. tribution within the target. Thus, the fluorescence decays to half its peak value at larger $z$ for increasing target size: $1.7,4.3$, and $6.3 \mathrm{~nm}$ for the quantum dot, nanorod, and sphere, respectively. Because of the convolution, the halfmaximum at $z=1.7 \mathrm{~nm}$ for the quantum dot represents an upper limit for the spatial confinement of the tipenhanced intensity profile. This value is several times smaller than previous measurements for either silicon [7] or metal $[4,6,10,14]$ tips.

The fluorescence count rate is enhanced by a factor of 19 for the quantum dot, a factor of 3 for the nanorod, and a factor of 4 for the $20 \mathrm{~nm}$ diameter fluorescent sphere. Previous measurements that used silicon tips showed less than a fivefold increase for quantum dots $[8,9]$ and less than a 50\% increase for $20 \mathrm{~nm}$ spheres [7]. Further, those experiments were complicated by interference effects that yielded a net suppression of the signal relative to the fluorescence background. An additional experiment used a modified aperture-type near-field tip and showed evidence of enhancement that was difficult to quantify [14].

Figure 2 plots each approach curve from Fig. 1 on a log$\log$ scale. Unity is subtracted from the vertical scales of Fig. 1 and the horizontal scales are offset by the fitting parameter $a$, whose physical relevance is discussed below.

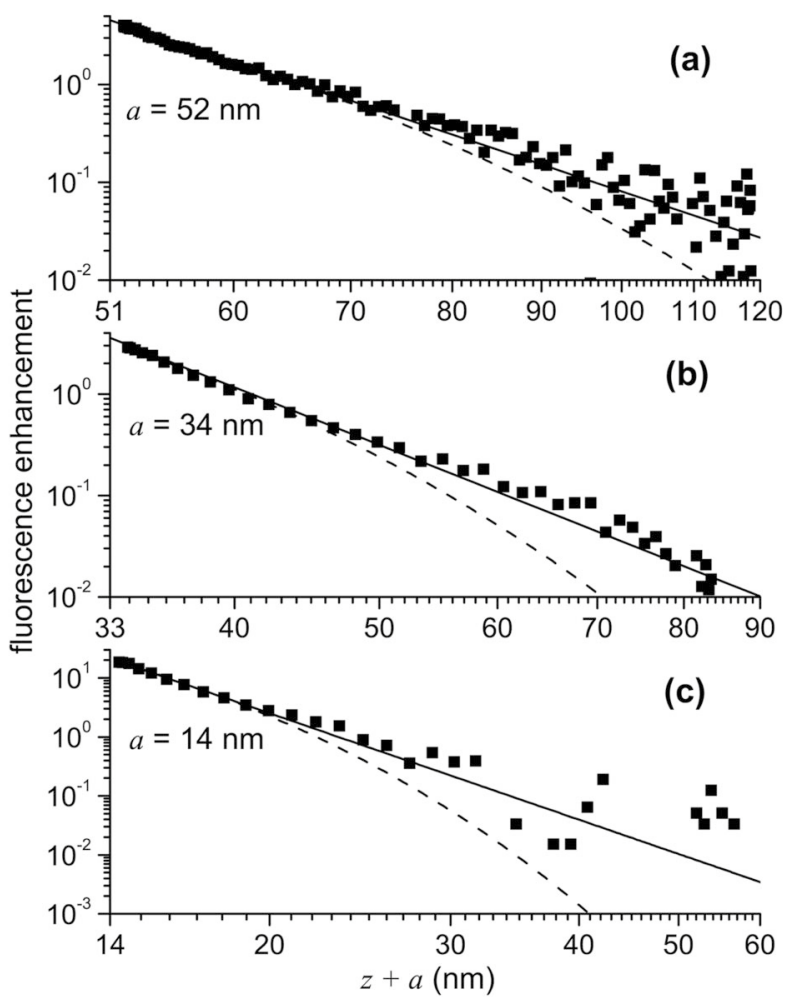

FIG. 2. Least-squares fit to the approach curve for (a) $20 \mathrm{~nm}$ diameter dye-doped sphere; (b) nanorod; and (c) spherical quantum dot. Fits to the power law of Eq. (1) (solid curves) and an exponential function (dashed curves) are shown with the data. The horizontal scales are offset by the fitted values of $a$, and unity is subtracted from the vertical scales. 
The linear appearance of the data on the log-log scale indicates a power-law decay, and the fluorescence enhancement $(\zeta)$ is fit to the function

$$
\zeta=\frac{F(z)}{F_{\infty}}-1=\kappa^{2}\left(\frac{a}{z+a}\right)^{6}
$$

where $F(z) / F_{\infty}$ is the detected fluorescence rate normalized to the background rate $\left(F_{\infty}\right)$, and $\kappa$ is the fieldenhancement factor. The solid curves show least-squares fits to Eq. (1) ( $\kappa, a$ free parameters) while the dashed curves are exponential decays. The data are clearly consistent with a power law, and deviate systematically from the best exponential fit. Previously, both power law [7,14] and exponential [4] decays were fit to experimental data with equal success. The precision of those experiments was insufficient to differentiate between various models because they did not probe the high-contrast region within several nanometers of tip-sample contact.

The measured $(z+a)^{-6}$ decay corresponds to the "near-field" term in the expression for the field intensity near a point dipole, where $z$ is the distance between the apex of the tip and the sample surface. Thus in the limit of an infinitesimally small target particle, the tip-enhanced field is equivalent to a dipole field whose singularity is located within the probe at a distance $a$ from the apex, where $a$ is the tip radius-of-curvature. For finite-sized target particles, the parameter $a$ is a measure of the degree of convolution between the intensity distribution and the excitation-probability distribution, and as expected the fitted values of $a$ increase for larger targets. For smaller targets, $a$ is converging to a value $(\sim 10 \mathrm{~nm})$ that is characteristic of the silicon probes used here. This is evidence that the field decay is indeed moderated only by the sharpness of the tip.

Because this technique utilizes a large probeoscillation amplitude, the fluorescence rate is modulated with maximum contrast, from the background level when the tip is $30-40 \mathrm{~nm}$ above the sample to the peak enhancement at tip-sample contact (Fig. 1). The tipenhanced intensity profile has no "far-field" component proportional to $r^{-2}$ (Fig. 2) and the corresponding fluorescence profile arises from the "near-field" intensity distribution only. Thus, the depth of fluorescence modulation that results from oscillation of the probe is decoupled from the far-field background and the near-field signal is easily extracted by subsequent demodulation.

A demodulated fluorescence image of a nanorod is shown in Fig. 3(a). The image was generated by raster scanning the AFM probe at a rate of 4 lines/s and then dividing each line into spatiotemporal pixels. These scan rates are at least 5 times faster than previous work for one-photon fluorescence [7-9] and 10 times faster for both two-photon fluorescence [6] and Raman scattering [4]. Pixel values are computed offline as the component of the photon-time-trace's Fourier power spectrum at the

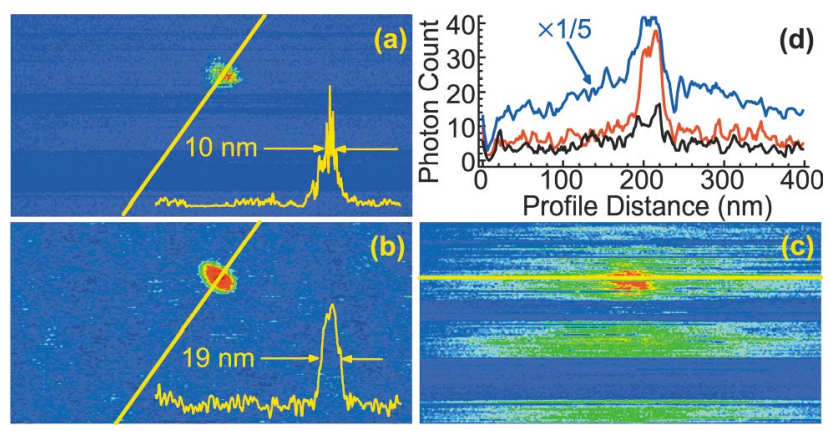

FIG. 3 (color). Tip-enhanced fluorescence image of a nanorod. (a) Fluorescence demodulation signal. (b) AFM probe height (5 nm at peak). (c) Total photon count. Insets in (a) and (b) show signal profiles along the designated axis of length $250 \mathrm{~nm}$. Blue curve in $(\mathrm{d})$ shows the total photon count $(\times 0.2)$ along the horizontal axis in (c), while red and black curves show the photon count within two ranges of tip-sample separation: $0<z<0.4 \mathrm{~nm}$ (red) and $2.5<z<4.5 \mathrm{~nm}$ (black). Field-of-view is $400 \times 200 \mathrm{~nm}$ : 256 lines of 1024 pixels each.

probe-oscillation frequency

$$
P=\left(\sum_{i} \sin \Delta_{i}\right)^{2}+\left(\sum_{i} \cos \Delta_{i}\right)^{2},
$$

where $\Delta_{i}$ are the measured phase delays and the summation is over all detected photons for the given pixel. When the probe is in the lateral vicinity of the target, the $\Delta_{i}$ are biased toward the phase value of tip-sample contact where the fluorescence rate is maximally enhanced. Otherwise, the $\Delta_{i}$ are uniformly distributed and $P$ vanishes to within the shot noise. Comparing Figs. 3(a) and 3(b) shows that the spatial resolution of the demodulated image (see below) is comparable to the tip sharpness and surpasses even the AFM resolution. Comparing Figs. 3(a) and 3(c) demonstrates the effectiveness of the analysis [Eq. (2)] in suppressing the fluorescence background.

Figure 3(d) shows three signal profiles along the horizontal axis indicated in Fig. 3(c) corresponding to the summation of photons over the entire probe-oscillationcycle (blue), and within a tip-sample separation range of $0<z<0.4 \mathrm{~nm}$ (red), and $2.5<z<4.5 \mathrm{~nm}$ (black). Here, horizontal profiles are chosen to avoid the regions of quantum-dot blinking [dark stripes in Fig. 3(c)]. As indicated on the figure, the data corresponding to the blue profile have been divided by a factor of 5 . Nearly $20 \%$ of the detected photons are emitted when the tip apex is within $0.4 \mathrm{~nm}$ of the sample surface (red curve) even though this corresponds to only $\sim 3 \%$ of the oscillation period. The black curve approximates the typical scanning conditions of previous ANSOM experiments which maintained a tip-sample gap roughly in this range [7-9]. Clearly, those conditions yield both inferior contrast and resolution compared to our technique.

The approach curve measurements (Figs. 1 and 2) suggest a straightforward approach for estimating the 


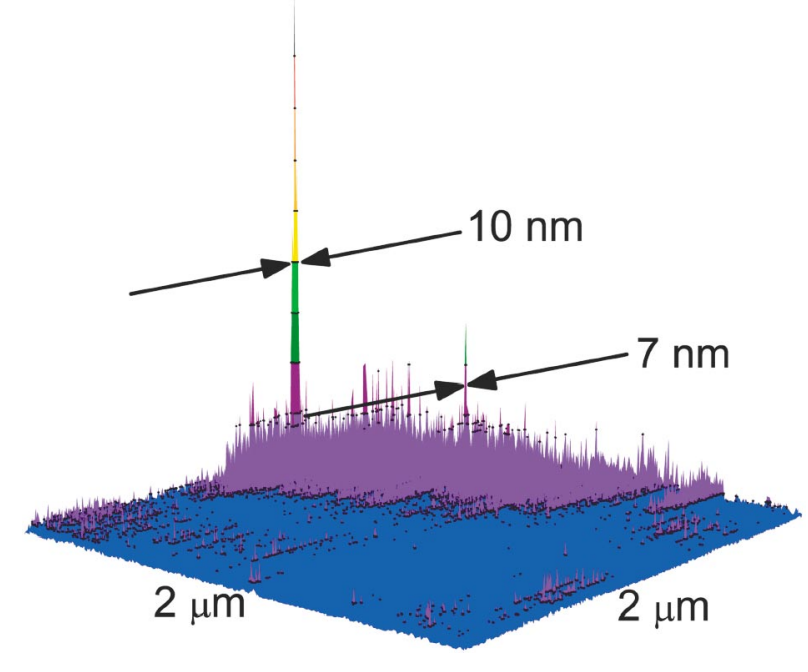

FIG. 4 (color). Tip-enhanced fluorescence image of quantum dots. The degree of contrast is emphasized by false-color relief. The image contains 512 lines of 1000 pixels each. The arrows indicate the measured FWHM for two quantum dots.

spatial resolution. The tip-enhanced field is modeled by a point dipole using a conservative estimate for tipcurvature $(a=14 \mathrm{~nm})$ as suggested by the quantum-dot approach curve in Fig. 2(c). A Monte Carlo simulation is then used to generate "mock" data from two hypothetical point sources separated by some distance and the analysis algorithm [Eq. (2)] is applied. The minimum resolvable separation between the point sources is then determined by applying the Sparrow criterion to the demodulated image, i.e., where the central dip between the two sources vanishes [24]. Use of the Sparrow rather than the Rayleigh criterion assures that the estimated resolution is independent of the particular moment calculated in Eq. (2). The simulations suggest a spatial resolution of 11-12 $\mathrm{nm}$ for the nanorod images shown in Fig. 3. Figure 4 shows a fluorescence demodulation image of spherical quantum dots in false-color relief. The arrows indicate the measured FWHM for two dots, and the model suggests a spatial resolution of $\sim 8 \mathrm{~nm}$ for the smaller one.

In conclusion, we made the first definitive measurement of the tip-enhanced optical field at the apex of a sharp probe and rigorously confirmed a dipolelike model. The technique developed for these measurements overcomes several major obstacles in ANSOM performance and led to the first one-photon fluorescence images with resolution below $10 \mathrm{~nm}$. In contrast to previous work, the tipenhanced excitation rate is maximized because the probe apex intermittently contacts the sample and thus the enhanced field is probed at atomic-scale distances from the apex. The intermittent tip-sample contact also induces modulation of the excitation rate; demodulating the resultant signal strongly suppresses the problematic far-field background and enables spatial resolution limited only by tip sharpness. The improvements in background suppression and spatial resolution will be even more acute for multiphoton processes (surface-enhanced Raman scattering; coherent anti-Stokes Raman scattering; two-photon fluorescence) compared to one-photon fluorescence, because the induced signal is then more strongly confined at the tip apex. In the future, it may be possible to image samples in a wet environment to measure dynamic processes in molecular-scale structural biology. Finally, it may also be possible to use carbon single-wall nanotubes attached to AFM probes $[25,26]$ to further improve spatial resolution.

We thank Delia Milliron, Ben Boussert, and Paul Alivisatos for the nanocrystals and acknowledge the financial support of Bruce Burrows and Pharmagenomix Inc. J. M. G. was partially supported by the Beckman Institute.

*Permanent address: University of UT, Department of Physics, 115 South 1400 East, Salt Lake City, UT 84112.

[1] F. Zenhausern, Y. Martin, and H. K. Wickramasinghe, Science 269, 1083 (1995).

[2] H. F. Hamann, A. Gallagher, and D. J. Nesbitt, Appl. Phys. Lett. 73, 1469 (1998).

[3] R. Hillenbrand and F. Keilmann, Appl. Phys. Lett. 80, 25 (2002).

[4] A. Hartschuh et al., Phys. Rev. Lett. 90, 095503 (2003).

[5] T. Ichimura et al., Phys. Rev. Lett. 92, 220801 (2004).

[6] E. J. Sánchez, L. Novotny, and X. Sunney Xie, Phys. Rev. Lett. 82, 4014 (1999).

[7] H. F. Hamann et al., J. Chem. Phys. 114, 8596 (2001).

[8] V.V. Protasenko et al., Opt. Commun. 210, 11 (2002).

[9] V.V. Protasenko, A. Gallagher, and D. J. Nesbitt, Opt. Commun. 233, 45 (2004).

[10] A. Kramer et al., Appl. Phys. Lett. 80, 1652 (2002).

[11] A. Bouhelier et al., Phys. Rev. Lett. 90, 013903 (2003).

[12] Y. Kawata, C. Xu, and W. Denk, J. Appl. Phys. 85, 1294 (1999).

[13] L. Novotny, J. Am. Ceram. Soc. 85, 1057 (2002).

[14] H. G. Frey et al., Appl. Phys. Lett. 81, 5030 (2002).

[15] L. Novotny, R. X. Bian, and X. S. Xie, Phys. Rev. Lett. 79, 645 (1997).

[16] J. Azoulay et al., Europhys. Lett. 51, 374 (2000).

[17] J. L. Bohn, D. J. Nesbitt, and A. Gallagher, J. Opt. Soc. Am. A 18, 2998 (2001).

[18] Y.C. Martin, H. F. Hamann, and H. K. Wickramasinghe, J. Appl. Phys. 89, 5774 (2001).

[19] B. Sick et al., J. Microsc. 202, 365 (2001).

[20] L. Aigouy et al., Opt. Lett. 24, 187 (1999).

[21] We used NanoDevices Multi75 (50-80 kHz oscillation frequency) and TAP300 $(250-350 \mathrm{kHz})$ cantilevers.

[22] R. G. Neuhauser et al., Phys. Rev. Lett. 85, 3301 (2000).

[23] M. Kuno et al., J. Chem. Phys. 112, 3117 (2000).

[24] E. Hecht, Optics (Addison-Wesley, Reading, 1990), 2nd ed.

[25] J. H. Hafner et al., J. Phys. Chem. B 105, 743 (2001).

[26] L. A. Wade et al., Nano Lett. 4, 725 (2004). 